\title{
Morphology of shock-accelerated multiphase flow: experiment and modeling
}

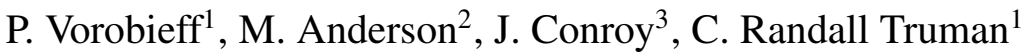 \\ \& S. Kumar ${ }^{4}$ \\ ${ }^{I}$ Department of Mechanical Engineering, The University of New Mexico, \\ Albuquerque, USA \\ ${ }^{2}$ Illinoisrocstar LLC, Champaign, Illinois, USA \\ ${ }^{3}$ Applied Research Associates, Albuquerque, New Mexico, USA \\ ${ }^{4}$ Department of Engineering, The University of Texas - Brownsville, \\ Brownsville, Texas, USA
}

\begin{abstract}
It was recently observed that vortices develop in multiphase media of non-uniform average density undergoing acceleration. This vortex formation is somewhat akin to vortex roll-up in gases of fluids (single-phase) due to Rayleigh-Taylor or Richtmyer-Meshkov instabilities. Differences in the underlying physics are negligible in the case of sustained modest acceleration (Rayleigh-Taylor instability), where conservation of momentum accounts for different velocities of volumes with different average densities. In the case of impulsive acceleration, the mechanism responsible for the multiphase analog of Richtmyer-Meshkov instability is peculiar to multiphase flow and is explained by post-acceleration interaction of the embedded phase (e.g., droplets) with the embedding phase (e.g., gas). Impulsive acceleration of a multiphase medium can also produce spatial rearrangement of the embedded particles or droplets in accordance with their size, noticeably altering the observed flow morphology. A careful numerical simulation explicitly accounting for the embedded phase behavior is required to faithfully reproduce the experimental results.
\end{abstract}




\section{Introduction}

Among the most physically important hydrodynamic instabilities are interfacial instabilities that develop on a density interface in the presence of acceleration. Stationary acceleration in the direction opposing the density gradient (if the acceleration is due to gravity, the heavy fluid is above the light fluid) produces Rayleigh-Taylor instability (RTI) [1-3]. The interface is stable to perturbations if the stationary acceleration is aligned with the density gradient. Impulsive acceleration (regardless of its orientation with respect to the density gradient) produces Richtmyer-Meshkov instability (RMI) [4, 5]. As both instabilities manifest themselves, vortices form on the interface, leading to growth of initial perturbations, formation of secondary instabilities (e.g., the shear-driven KelvinHelmholtz instability), and ultimately transition to turbulence that causes the interface to become well-mixed. For problems involving RTI, a gravity field is most likely to provide the acceleration. For problems where RMI is important, it often arises due to shock passage through the medium, which means that compressibility and thermal effects also must be considered. Both instabilities, however, can be predicted with theoretical analysis of ideal fluid flow.

The variety of problems to which shock-driven interface evolution is essential is quite wide, ranging from astrophysics and planetary physics to engineering applications, the latter including inertial confinement fusion (ICF) [6] and fuel combustion in power plants, engines, etc. [7]. Moreover, in many of these problems the shock does not merely propagate through a variable-density mixture of gases/plasmas: the medium is in fact multiphase, with non-gaseous inclusions (fluid droplets or solid particles) making an important contribution to the density field. In the latter case, vortices were recently found to form even when the flow field does not contain gases of different densities [8,9], but the average density of the medium varies because of the contribution of the droplet or particle phase, with particles/droplets initially seeding the flow volume non-uniformly. This phenomenon is referred to as particle lag instability (PLI). The initial investigations found PLI development to be strongly influenced both by the particle/droplet size distribution (which affects post-shock particle lag) and by the effective Atwood number of the mixture

$$
A=\left(\rho_{1}-\rho_{2}\right) /\left(\rho_{1}+\rho_{2}\right),
$$

where $\rho_{1}$ is the average density of the seeded gas and $\rho_{2}$ is the density of the unseeded gas. The effective Atwood number influences the instability growth in a fashion similar to that in which the flow driven by RMI is influenced by the traditionally-defined Atwood number based on the density difference of gases on the interface. In that case, $\rho_{1}$ and $\rho_{2}$ in Eq. 1 would correspond to the densities of gases on each side of the gas-gas interface undergoing shock acceleration.

Here we present a numerical investigation of the particle lag instability that uses comparison with experimental studies both for code validation and to draw inferences about the likely post-shock droplet size distribution in experiment, which has not yet been measured directly. 


\section{Experiments}

The experiments are conducted at the University of New Mexico shock tube facility (Fig. 1). The driver section of a horizontally oriented shock tube is pressurized with helium to a prescribed pressure. Subsequently a solenoid-driven puncturer ruptures the diaphragm that separates the driver section from the driven section, initially filled with air at rest and at ambient pressure. This releases a shock wave into the driven section. The shock wave traverses the driven section (which has a square $7.62 \times 7.62 \mathrm{~cm}$ cross-section) and reaches the transparent test section, where air seeded with micron-sized propylene glycol droplets (less than $5 \%$ by volume) is injected vertically through the top wall, forming a nearly two-dimensional column.

The effective Atwood number (Eq. 1) characterizing the experiment is measured to be about 0.03 , and the range of Mach numbers $M=V / a$ ( $V$ being the shock front speed and $a$ - the speed of sound in uncompressed quiescent air upstream of the shock) is varied from 1.2 to 2.1. A pair of double-pulsed Nd:Yag lasers with an attached combination of a cylindrical and a spherical lens produces a light sheet (labeled "laser sheet" in Fig. 1) that illuminates a horizontal section of the flow field at the center plane of the shock tube. Resulting images are captured with an intensified, high framing speed multiple-CCD camera. Each laser pulse duration is about $5 \mathrm{~ns}$, and a sequence of up to four images of the evolving flow can be captured during a single experiment. The limitation of four exposures per experiment is imposed by the laser visualization system, where each of the two double-pulsed lasers can only be fired once during an experiment (the laser repetition rate is $15 \mathrm{~Hz}$, while a typical duration of the experiment is less than $1 \mathrm{~ms}$ ). Flow visualization relies on Mie scattering off the glycol droplets. The droplet size could be inferred from the scattering intensity and estimated from their settling time in quiescent air, ruling out the presence of droplets larger than several microns.

Experiments with the same initial conditions and Mach numbers are repeated with different exposure timings to capture the details of the flow evolution. Thus the flow morphology, starting with the pre-shock initial conditions, and growth rates of the dominant features of the flow (e.g., the counter-rotating vortex columns) are known through the duration of the experiment. The latter is Mach number-dependent, and is determined by how long it takes from the moment of the shock arrival at the initial conditions to the time when the flow volume containing the seeding particles leaves the test section.

\section{Numerical model}

In earlier work [9], two computational approaches were considered. The first used an assumption that the seeded gas could be modeled as a single-phase medium with all the properties except density considered to be the same as the properties of unseeded air, and the density altered to account for the added mass of the glycol droplets. This approach produced satisfactory results in terms of reproducing the 

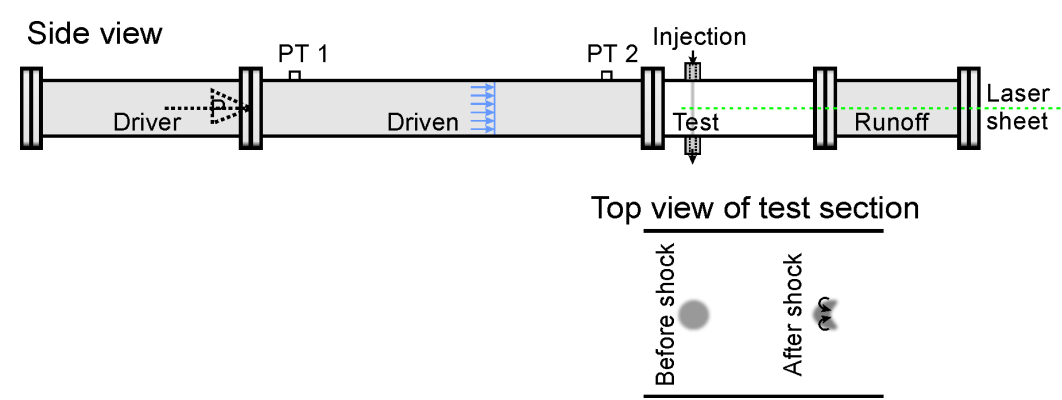

Figure 1: Schematic of experimental setup. Top: side view. The pressurized driver section (left) is separated from the driven section by a diaphragm that is ruptured by a puncturer (dashed lines, labeled P). As the shock (schematically shown) propagates through the driven section, its progress is monitored by two pressure transducers (PT1 and PT2). Glycol droplets suspended in air are injected into the text section vertically, forming a seeded cylindrical column. The flow is illuminated with a horizontal laser sheet through an optical window in the end of the runoff section. Bottom: close-up of the laser-illuminated flow visualization plane in the test section as seen from above, showing the cross-section of the initial conditions and a dynamic image with a counter-rotating vortex pair.

counter-rotating vortices observed in experiment, but could not reproduce subtle morphological features. The second computational method took into account the post-shock interaction between the gas and the particles or droplets, yielding more promising results. Thus in the present work we concentrate on the second approach.

The modeling was performed using SHAMRC (Second-order Hydrodynamic Automatic Mesh Refinement Code) [10]. It is a two- and three-dimensional code that solves the conservation equations of fluid motion on an Eulerian grid, using an operator-split explicit time-marching scheme that is second-order accurate in both space and time. Each of the conservation equations (mass, momentum, energy) is divided into Lagrangian and Eulerian terms, and the solution is split into two corresponding phases. During the first (Lagrangian) phase, the conservation equations are solved in a Lagrangian frame of reference, moving with the flow for half a time step. Then any energy transfer effects (due to chemical reactions, radiation, etc.) are applied. The results of the first phase are then remapped onto a fixed (Eulerian) mesh, and the second phase commences. The latter takes the form of an advection calculation which considers the fluxes of the hydrodynamic variables. Subsequently the effects due to the presence of particles or drops in the flow are considered. Particles can exchange mass, momentum, and energy with the fluid. For each computational particle, properties such as mass, density, 
position, velocity, etc. are tracked. In computing the particle interaction with the surrounding fluid, each such particle is considered as a representative from a cloud of a prescribed large number of particles with identical properties. This makes it possible to model large quantities of particles with a modest computational effort. The model (referred to as MIP, or massive interactive particles) is limited in the sense that it cannot account for particle collisions or pressure effects due to the presence of the particulate phase, restricting the code to modeling suspensions where the embedding fluid phase occupies most of the volume, as is the case in our experiments. Accounting for the particle interactions with the flow completes the time step of the code.

In the modeling described in the following section, the computational domain was limited to two spatial dimensions, modeling the flow in a horizontal plane in the middle of the test section of the shock tube. The Cartesian mesh used for modeling had a fixed step of $0.005 \mathrm{~cm}$ in both $x$ (streamwise) and $y$ (normal to streamwise) directions. The mesh extended from the origin (at the center of shock tube cross-section) to the test section walls in the $y$ direction, a distance of $3.81 \mathrm{~cm}$. The $x$ (streamwise) extent of the computational domain was set to $40 \mathrm{~cm}$ so that the flow evolution could be observed in the same region that was visualized in the experiments before being swept from the grid. Symmetry with respect to the $x$-axis was assumed to model only half of the domain $(y \geq 0)$, reducing the total number of zones required to 6 million.

To generate the shock waves in the simulations, high pressure and temperature air at a specific velocity was placed upstream of the initial conditions. To maintain steady flow, these conditions were also fed in from the left boundary. The RankineHugoniot relations for a calorically perfect gas were used to determine the gas properties required to generate the Mach number matching the experiment.

\section{Results and discussion}

Figure 2 shows the evolution of the particle lag instability (PLI) from nearlycylindrical initial conditions to a pair of counter-rotating vortex columns with a prominent "tail" of droplets trailing behind. The formation of this "tail" is one of the morphological features distinguishing the multiphase analog of RMI from RMI proper that develops near a gas-gas density interface. After shock passage, all droplets, whose density is much higher than that of air, lag behind the accelerated and compressed gaseous phase. The time it takes for a droplet to equilibrate its velocity with the surrounding air is shorter for smaller droplets and longer for larger and more massive droplets, which form the "tail." Due to this momentum exchange, the air volume containing most of the droplets reaches an equilibrium velocity which is below that of the surrounding droplet-free air. The resulting shear leads to vortex roll-up.

From experimental images, it is thus apparent that the droplets are non-uniform in size. However, the droplet sizes cannot be measured directly, as the droplets are much smaller than the pixel resolution of the camera (about $16 \mu \mathrm{m}$ per pixel). Moreover, their micron- to sub-micron sizes would also be hard to resolve 

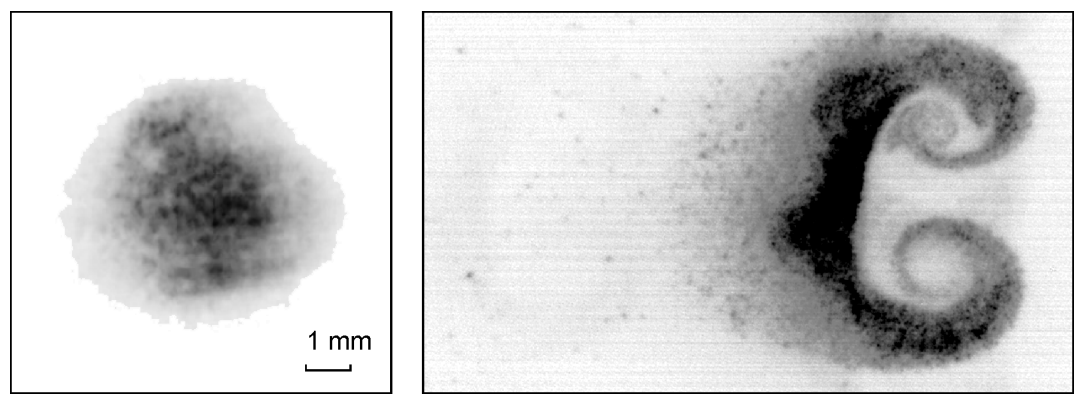

Figure 2: Results of experimental visualization of a shock-accelerated, glycol droplet-seeded air column at $M \sim 1$.7. Images are inverted, so that darker areas correspond to higher Mie scattering intensity off droplets. Left: cross-section of initial conditions. Right: dynamic image at $910 \mu \mathrm{s}$ after shock acceleration of the column. Note the counter-rotating vortex pair and the "tail" of droplets. Flow direction is from left to right.

optically because of diffraction limitations (the wavelength of the laser light used for illumination is $532 \mathrm{~nm}$ ). It is also known from direct measurements that the density of ambient unseeded air in our laboratory was $1.05 \mathrm{~kg} / \mathrm{m}^{3}$ (lower than that at standard atmospheric pressure due to elevation), while the average density of the air-droplet mix was $1.12 \mathrm{~kg} / \mathrm{m}^{3}$.

For modeling, this difference between the average densities had to be matched by adding an appropriate volume fraction of glycol droplets (density $1036 \mathrm{~kg} / \mathrm{m}^{3}$ ) to air. The same volume fraction would be accounted for by fewer larger droplets or by more smaller droplets. Initially, several monodisperse droplet distributions were considered, with droplet sizes $0.5,0.7,3,5$, and 10 microns. For the largest $(10 \mu \mathrm{m})$ droplets, vortex formation was nowhere near as prominent in numerics as in experiment, while the smallest computational droplets were advected into the vortex cores more readily than the droplets observed in experiment. The computational droplet size matching the observed morphology and the instability growth rate most closely was $3 \mu \mathrm{m}$. However, because of particle monodispersity, the "tail" feature of the flow morphology could not be reproduced.

In an attempt to match the morphology of the experimental PLI results even better, we then considered the effect of variation in droplet size by assuming that these sizes followed a log-normal distribution with a mean of $3 \mu \mathrm{m}$. We then performed calculations for such size distributions with a standard deviation of 0.2 , 0.5 , and $1.0 \mu \mathrm{m}$. Figure 3 shows the results of the modeling, with the standard deviation increasing column by column from left to right, the leftmost sequence of images corresponding to the monodisperse case. The evolution of the particle tail size in experiments is best matched by the distribution with a standard deviation $0.5 \mu \mathrm{m}$. 


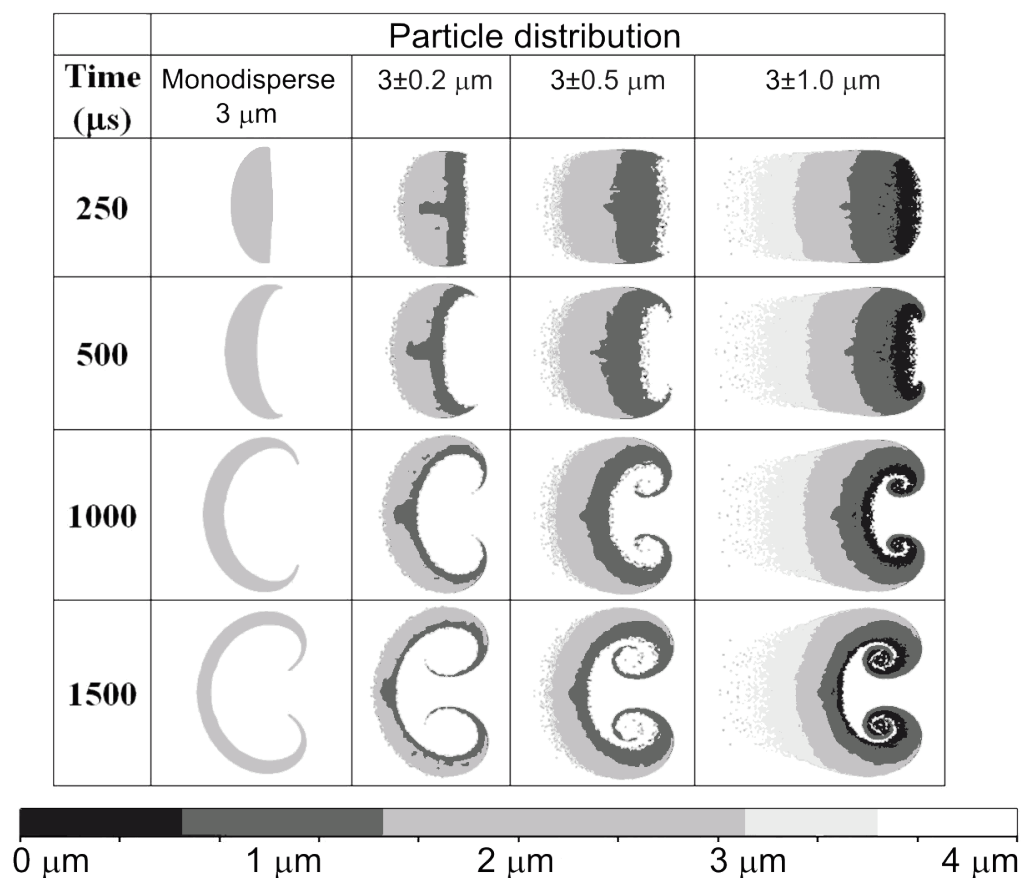

Figure 3: Comparison of results of numerical modeling $(M=1.7)$ with different particle size distributions. A vertical column of images corresponds to each distribution. The first column shows the results for monodisperse 3- $\mu$ m particles. In subsequent column labels, numbers after \pm denote the standard deviation of the corresponding lognornal distribution of particle sizes. Row labels denote time in $\mu$ s after shock acceleration of the initial conditions.

\section{Conclusion}

A carefully performed numerical modeling of shock-accelerated multiphase flow with SHAMRC code can reproduce the flow morphology of an experimentally produced instability and quantitatively match the growth rates of the flow features. For a full validation of the model used to represent advection of particles or droplets by the flow, future experiments should include direct measurement of droplet sizes.

The study also sheds some light on the development of the particle lag instability (PLI). Recent papers describe its formation as the result of post-shock momentum exchange between slower-moving particles (droplets) and shock-accelerated gas surrounding them. As the result, in the gas volume containing the embedded phase, some average equilibrium velocity is reached, with particles/droplets no longer having a relative velocity with respect to the gas surrounding them. This velocity 
is lower than that of the unseeding phase, which leads to shear and vortex rollup. This physical mechanism is limited to a certain range of embedded phase sizes. Consider two limit cases, in both of which the embedded phase occupies the same volume fraction. In the first case (particles/droplets are very small, close to molecular limit), the physical mechanism responsible for PLI is not realized, and Richtmyer-Meshkov instability develops instead, effectively between two gases of different densities. In the second limit case (all the added mass is contained in one big droplet), a single wake behind the droplet develops, but no velocity equilibrium with the embedding gas is reached during the relatively short time interval of interest. Numerical modeling supports this notion, with smaller computational droplet sizes manifesting flow morphologies similar to that of RMI, and with the largest size of droplets in numerics showing no discernible vortex roll-up (and thus no PLI).

\section{Acknowledgement}

This research is supported by the US National Nuclear Security Administration (NNSA) grant DE-FG52-10NA29648.

\section{References}

[1] Strutt), L.R.J., Investigation of the character of the equilibrium of an incompressible heavy fluid of variable density. Proceedings of the London Mathematical Society, 14, pp. 170- 177, 1883.

[2] Taylor, G., The instability of liquid surfaces when accelerated in a direction perpendicular to their planes. Proceedings of the Royal Society of London Series A, Mathematical and Physical Sciences, 201(1065), pp. 192-196, 1950.

[3] Lewis, D., The instability of liquid surfaces when accelerated in a direction perpendicular to their planes. II. Proceedings of the Royal Society of London Series A, Mathematical and Physical Sciences, 202(1068), pp. 81-96, 1950.

[4] Richtmyer, R.D., Taylor instability in shock acceleration of compressible fluids. Communications in Pure and Applied Mathematics, 13(2), pp. 297319, 1960.

[5] Meshkov, E.E., Instability of the interface of two gases accelerated by a shockwave. Izv AN SSSR, Mekh Zhidk Gaza, 4(5), pp. 151-157, 1969.

[6] Youngs, D., Numerical simulation of mixing by Rayleigh-Taylor and Richtmyer-Meshkov instabilities. Laser and Particle Beams, 12, pp. 725750, 1994.

[7] Wang, C., Gu, Z., Dong, R., Zhang, L., Jia, H. \& Shi, H., Numerical simulation on detonation formation by shock/flame interaction. 28th International Symposium on Shock Waves, ed. K. Kontis, Springer Berlin Heidelberg, pp. 301-306, 2012. 
[8] Vorobieff, P., Anderson, M., Conroy, J., White, R. \& Truman, C.R., Vortex formation in a shock-accelerated gas induced by particle seeding. Physical Review Letters, 106, p. 184503, 2011.

[9] Vorobieff, P., Anderson, M., Conroy, J., White, R., Truman, C. \& Kumar, S., Analogues of Rayleigh-Taylor and Richtmyer-Meshkov instabilities in flows with nonuniform particle and droplet seeding. Computational Methods in Multiphase Flow VI, eds AA Mammoli, CA Brebbia, WIT Press, Southampton, UK, pp. 17-28, 2011.

[10] Crepeau, J., Needham, C.E. \& Hikida, S., Second Order Hydrodynamic Automatic Mesh Refinement Code (SHAMRC): Volume I, Methodology. Technical report, Applied Research Associates, Inc., 2001. 